\title{
Mutations in the C-Terminal Region of the HIV-1 Reverse Transcriptase and their Correlation with Drug Resistance Associated Mutations and Antiviral Treatment
}

\author{
I. Michels ${ }^{1}$, S. Staszewski², L. Gürtler ${ }^{1}$, G. Nisius², A. Müller ${ }^{3}$, L. Locher ${ }^{4}$, H.-W. Doerr ${ }^{1}$, \\ M. Stürmer ${ }^{1}$ \\ ${ }^{1}$ J.W.Goethe University Hospital, Institute for Medical Virology, Frankfurt/Main, \\ ${ }^{2}$ J.W.Goethe University Hospital, Medical HIV Treatment and Research Unit, Department of Internal Medicine II, Frankfurt/Main, \\ ${ }^{3}$ Infektiologikum Frankfurt-Sachsenhausen, Frankfurt/Main, \\ ${ }^{4}$ Infektiologikum Frankfurt-City, Frankfurt/Main, Germany
}

\begin{abstract}
Objective: Replication of HIV-1 after cell entry is essentially dependent on the reverse transcriptase (RT). Antiretroviral drugs impairing the function of the RT currently aim at the polymerase subunit. One reason for failure of antiretroviral treatment is the evolvement of resistance-associated mutations in the viral genome. For RT inhibitors, almost all identified mutations are located within the polymerase; therefore, general genotyping confines to investigate this subunit. Recently several studies have shown that substitutions within the $\mathrm{RNase} \mathrm{H}$ and the connection domain increase antiviral drug-resistance in vitro, and some of them are present in patient isolates.

Aim: The aim of the present study was to investigate the prevalence of these substitutions and their association with mutations in the polymerase domain arising during antiretroviral treatment.

Material and Methods: We performed genotypic analyzes on seventy-four virus isolates derived from treated and untreated patients, followed at the HIV Centre of the Johann Wolfgang Goethe University Hospital (Frankfurt/Main, Germany). We subsequently analysed the different substitutions in the c-terminal region to evaluate whether there were associations with each other, n-terminal substitutions or with antiretroviral treatment.

Results: We identified several primer grip substitutions, but almost all of them were located in the connection domain. This is consistent with other in-vivo studies, in which especially the primer grip residues located in the RNase $\mathrm{H}$ were unvaried. Furthermore, we identified other substitutions in the connection domain and in the RNase H. Especially E399D seemed to be associated with an antiretroviral treatment and $\mathrm{N}$-terminal resistance-delivering mutations.

Conclusion: Some of the identified substitutions were associated with antiviral treatment and drug resistanceassociated mutations. Due to the low prevalence of Cterminal mutations and as only a few of them could be associated with antiviral treatment and N-terminal resistance-delivering mutations, we would not recommend routinely testing of the C-terminal RT region.
\end{abstract}

Key words: antiretroviral therapy, genotypic resistance, reverse transcriptase, polymerase, RNase H, connection domain

\section{INTRODUCTION}

The development of antiretroviral therapy has reduced the mortality and morbidity of HIV. Several drugs are available for the treatment of HIV, many of which are inhibitors of the reverse transcriptase (RT) [1].

Currently, two different classes of RT-Inhibitors are available, non-nucleoside reverse transcriptase inhibitors (NNRTIs) and nucleoside reverse transcriptase inhibitors (NRTIs).

The RT has a high error rate; combined with the high replication capacity of HIV (1-6 hours), mutations resulting in antiviral drug-resistance can evolve easily. Since NRTIs block the polymerase subunit of the RT, NRTI-resistance delivering mutations are mainly expected to be located there. The commercially available genotyping systems confine to investigate this subunit.

NRTI-resistance can either be caused by an enhanced excision or selectivity of the RT [1-4]. Recently it has been suggested that $\mathrm{C}$-terminal mutations might increase the timeframe for NRTI-excision by decreasing the RNase $\mathrm{H}$ activity $[3,5]$. Genotypic and phenotypic analyses of NRTI-experienced RTs showed that mutations in the connection domain (E312Q, G335C/D, N348I, A360I/V, V365I and A376S) were able to increase NRTI- resistance [6]. Nikolenko et al. proposed that such mutations might affect the RNase $\mathrm{H}$ primer grip, resulting in inefficient and unspecific template-cleavage [7].

If C-terminal mutations prolong the timeframe for nucleotide excision, additional mutations enhancing NRTI-excision might lead to a synergistic increase in antiretroviral drug-resistance. Accordingly almost all mutations described in previous studies could not enhance azidothymidine (AZT)-resistance significantly, unless they were combined with tymidinanalogue-resistance associated mutations (TAMs) [7, 8]. In the meantime several in vivo studies highlighting the need 
for further investigation of the $\mathrm{C}$-terminal region have been published [9-12].

The aim of the present study was to investigate the prevalence of C-terminal substitutions and to analyse if there are any associations with $\mathrm{N}$-terminal resistance-delivering mutations or antiretroviral treatment.

\section{MATERIAL AND METHODS}

\section{PATIENTS}

Seventy-four viral sequences from HIV-1 infected patients followed at the HIV Centre of the Johann Wolfgang Goethe University Hospital (Frankfurt/Main, Germany) from October 2005 to August 2007 were included in this study. The sequences were randomly chosen, but only viral isolates with a subtype B according to the pol gene region were integrated in this study. Furthermore, sequences of patients who were in a treatment interruption (TI) longer than six months at the time of sample taking were excluded.

The median viral load was 4900 copies/ml (range 306-10.000.000 copies/ml).

\section{Genotypic Resistance Testing}

We extracted HIV-1 RNA from one ml of EDTAplasma using the QIAamp ${ }^{\circledR}$ Viral RNA Mini Kit (Qiagen, Hilden) according to the procedure recommended by the manufacturer.

The N-terminal region was amplified as previously described encompassing the polymerase region between codons 1 and 340 [13-15]. A semi-nested RT PCR was performed for the amplification of the Cterminal region analyzing codons 340-560. The QIAGEN® OneStep RT-PCR Kit (Qiagen, Hilden) was applied for the outer PCR, while the inner PCR was performed using the Taq DNA Polymerase and Q-Solution Kit (Qiagen, Hilden). The following primers were used for RT-PCR (position according to HXB2): Outer PCR Forward: 5'-ggACATATCAAAT'T'TAT CAAgARCCAT-3' (Tib Molbiol, Berlin); Outer PCR Reverse: 5'-CACTRgCYACATgAACTgCTACCA-3' (Tib Molbiol, Berlin); Inner PCR Forward: 5'-ggA CATATCAAATTTATCAAgARCCAT-3' (Tib Molbiol, Berlin); Inner PCR Reverse: 5'-TAgCTgCCATATTC CKggRCTAC-3' ('Tib Molbiol, Berlin).

The PCR product was sequenced using an in-house sequencing assay and the primers mentioned above. For purification of the sequencing product, the DyeEx 2.0 Spin Kit was applied. Genotyping was performed with a 3100 Avant Genetic Analyzer (ABI Prism, Darmstadt). The sequences were analyzed using the SeqMan Pro software (DNASTAR, Inc., Konstanz).

Mutations were reported as amino acid changes with respect to the sequence of the wild-type virus HXB2. Mixtures were considered as mutated.

\section{Statistical Methods}

All statistic calculations were done with the SPSS Software (Version 16.0). Exact Fisher's test was used to compare the prevalence of substitutions in the C-terminal region of treatment-naïve patients and pre-treated patients. We also used Fisher's test to find associa- tions between these substitutions and the frequency of TAMs, nucleoside analogue mutations (NAMs) and NNRTI-resistance-associated mutations. For all tests differences with a $\mathrm{p}<0.05$ were considered to be statistical significant.

\section{RESULTS}

\section{ANTIRETROVIRAL TrEatMENT}

25 samples were derived from therapy-naïve and 49 samples from pre-treated patients. Of these, 43/49 were derived from patients with AZT-medication in their antiretroviral history. Twenty-seven sequences were derived from patients taking NRTIs at the time of the sample collection, 15 received AZT, 18 received 3TC and three received FTC (see Table 1). The median number of NRTIs received by patients treated at the time of sample drawing was two (range 0-5), while the median number of protease inhibitors (PIs) was zero (range 0-3). Thirteen samples and derived sequences were collected from patients in treatment interruption (TI) with a median duration of four months (range 1-6 months).

\section{N-terminal Resistance-Associated Mutations}

Twenty-seven of the seventy-four analyzed sequences contained resistance-associated mutations (see Table 1). Twenty-four contained TAMs (M41L, D67N, K70R, L210W, T215YF and K219QE). The median number of TAMs was three (range 1-4). Sixteen contained additional NRTI-resistance associated mutations (NAMs), e.g. K70E, L74VI, V75T, Y115F and the M184V mutation, which was present in all 16 sequences. Eleven sequences contained NNRTI-resistance associated mutations (K103N, V179F, Y181C, Y188L and G190A).

\section{Amino Acid Heterogeneity in the C-Terminal REGION}

Seventy-four positions in the C-terminal part of the HIV-1 RT that have previously been implicated in NRTI resistance were analyzed in this study. However, we could not evaluate some sequences completely, which is why there is insufficient data for some positions. The following positions were conserved among the treatment-naive and pre-treated patients: Y318, H361, W401, F416, Q428, F440, A446, R448, D471, T473, N474, Q475, T477, Q500, Y501, I505, H539, V548 and D549. Only position 318, 361, 401 and 416 are part of the connection region; the other positions are located in the RNase $\mathrm{H}$ [8]. 55/74 examined positions $(74 \%)$ showed amino acid heterogeneity. These positions were mutated among the pre-treated, the treatment-naïve patients or both (see Table 2).

\section{Association of Mutated Amino Acids in the C- TERMINAL REgION WITH ANTIRETROVIRAL TREATMENT}

Substitutions at three positions (369, 399 and 554) were associated with an AZT-treatment six months prior to sample taking. Some Substitutions (395, 520 
Table 1. Baseline characteristics of the 49 pretreated viral isolates.

Characteristic Value

Age, median years (IQR)

Plasma HIV-1 RNA level, median copies/ml (IQR)

Previous antiretroviral therapy, median months (IQR)

Treatment interruption (TI) at time of sample-taking

$$
\begin{aligned}
& \text { Yes } \\
& \text { No } \\
& \text { Duration of TI, median months (IQR) }
\end{aligned}
$$

NRTI used in antiretroviral treatment at time of sample taking

Yes

No

Prior mono nucleoside treatment

Yes

No

Prior dual nucleoside treatment

Yes

No

3TC used in antiretroviral treatment at time of sample-taking

Yes

No

FTC used in antiretroviral treatment at time of sample-taking Yes

No

NNRTI used in antiretroviral treatment at time of sample taking

Yes

No

Prior NNRTI-treatment

Yes

No

PI used in antiretroviral treatment at time of sample taking

Yes

No

Prior PI-treatment

Yes

No

Sequences containing TAMs

TAMs, median no. (IQR)

M184V mutation present

Yes

No

Sequences containing NNRTI resistance delivering mutations

NNRIT resistance delivering mutations, median no. (IQR)
44 (23-80)

4900 (range 306-10.000.000)

124 (range 1-193)

$13(26,5)$

$36(73,5)$

4 (1-6)

$27(55,1)$

$22(44,9)$

$20(40,8)$

$29(59,2)$

$28(57,1)$

$21(42,9)$

$18(36,7)$

$31(63,3)$

$3(6,1)$

$46(93,9)$

$3(6,1)$

$46(93,9)$

$36(73,5)$

$13(26,5)$

$21(42,9)$

$28(57,1)$

$45(91,8)$

$4(8,2)$

$24(49)$

$3(1-4)$

$16(32,7)$

$33(67,3)$

$11(22,4)$

$1(1-3)$

NOTE. Data are no. (\%) of patients, unless otherwise specified. IQR, interquartile range, 3TC, 2',3'-dideoxy-3'-thiacytidine. FTC, 2',3'-Didesoxy-5-fluor-3'-thiacytidin.

and 524) showed the tendency to be associated with an AZT-treatment six month prior to sample taking. The only substitutions associated with a current antiretroviral therapy and a current NRTI-treatment were located at position 399 (see Table 3). Additionally mutations at position 524 and 554 were associated with a current AZT-treatment. Substitutions at position 554 also showed the tendency to be associated with a current NRTI-treatment and an AZT-treatment any time prior to sample taking. Substitutions at position 520 showed the tendency to be associated with a current antiretroviral treatment.

\section{Association of Mutated Amino Acids in the \\ C-TERMINAL REgION WITH RESISTANCE-DELIVERING N-TERminal Mutations}

Several C-terminal substitutions showed associations to N-terminal resistance-associated mutations (see Table 4). 
Table 2. Positions and frequencies of selected C-terminal mutations in sequences of viral isolate from HIV-1 infected patients. Amino acids in bold are only present in sequences derived from pre-treated patients $\left(^{*}\right)$.

\begin{tabular}{|c|c|c|c|c|c|c|}
\hline & \multirow[b]{2}{*}{$\begin{array}{l}\text { HXB2- } \\
\text { Sequence }\end{array}$} & \multirow[b]{2}{*}{$\begin{array}{l}\text { Substitutions in } \\
\text { total }(\%)\end{array}$} & \multicolumn{2}{|c|}{ therapy-naïve patients } & \multicolumn{2}{|c|}{ pre-treated patients } \\
\hline & & & Substitutions & Total $(\%)$ & Substitutions & Total (\%) \\
\hline 369 & $\mathrm{~T}$ & $5(8 \%)$ & $\mathrm{P}$ & $1(4 \%)$ & AIV & $4(10 \%)$ \\
\hline 395 & K & $9(13 \%)$ & $\mathrm{R}$ & $2(8 \%)$ & $\mathrm{R}$ & $7(15 \%)$ \\
\hline 399 & $\mathrm{E}$ & $15(21 \%)$ & $\mathrm{D}$ & $2(8 \%)$ & DGK & $13(29 \%)$ \\
\hline 520 & Q & $4(5 \%)$ & None & 0 & EHK & $4(8 \%)$ \\
\hline 524 & Q & $10(14 \%)$ & $\mathrm{E}$ & $2(8 \%)$ & EK & $8(16 \%)$ \\
\hline 554 & A & $37(51 \%)$ & NST & $10(40 \%)$ & NST & $26(54 \%)$ \\
\hline
\end{tabular}

* Only the Positions and frequencies of C-terminal mutations that are either statistical associated or showing the tendency to be associated with antiretroviral treatment are shown

Table 3. Association of selected C-terminal mutations with antiretroviral treatment (*).

\begin{tabular}{cccccc}
\hline $\begin{array}{c}\text { Position } \\
\text { according to } \\
\text { HXB2 }(* 2)\end{array}$ & $\begin{array}{c}\text { Current } \\
\text { antiretroviral } \\
\text { treatment [p=] }\end{array}$ & $\begin{array}{c}\text { Current NRTI- } \\
\text { treatment [p=] }\end{array}$ & $\begin{array}{c}\text { Current AZT- } \\
\text { treatment [p=] }\end{array}$ & $\begin{array}{c}\text { Prior AZT- } \\
\text { treatment [p=] }\end{array}$ & $\begin{array}{c}\text { AZT- } \\
\text { treatment } \\
\text { six months } \\
\text { prior to } \\
\text { sample- } \\
\text { taking [p=] }\end{array}$ \\
\hline 369 & 0.173 & 0.318 & 1 & 0.380 & 0.015 \\
395 & 0.154 & 0.481 & 0.670 & 0.283 & 0.054 \\
399 & 0.007 & 0.012 & 0.455 & 0.075 & 0.019 \\
520 & 0.051 & 0.134 & 1 & 0.142 & 0.086 \\
524 & 0.185 & 0.156 & 0.024 & 0.731 & 0.061 \\
554 & 0.816 & 0.052 & 0.046 & 0.077 & 0.024 \\
\hline
\end{tabular}

* Only the p-values of mutations that are either statistical associated or showing the tendency to be associated with antiretroviral treatment are shown

*2 All replacements that occurred at the indicated sites were included in the statistic analysis

Table 4. Association of selected C-terminal mutations with $\mathrm{N}$-terminal resistance-associated mutations $\left(^{*}\right)$

\begin{tabular}{cccc}
\hline $\begin{array}{c}\text { Position } \\
\begin{array}{c}\text { ccording to }) \\
\text { HXB2 }(* 2\end{array}\end{array}$ & $\begin{array}{c}\text { Association of C-terminal } \\
\text { mutations with TAMs } \\
{[\mathrm{p}=]}\end{array}$ & $\begin{array}{c}\text { Association of C-terminal } \\
\text { mutations with NAMs } \\
{[\mathrm{p}=]}\end{array}$ & $\begin{array}{c}\text { Association of C-terminal mutations } \\
\text { with NNRTI-resistance-delivering } \\
\text { mutations [p=] }\end{array}$ \\
\hline 358 & 0.095 & 0.608 & 0.113 \\
369 & 0.014 & 0.008 & 0.579 \\
395 & 0.002 & 0.043 & 0.112 \\
399 & $<0.001$ & 0.003 & 0.205 \\
431 & 0.139 & 0.675 & 0.024 \\
451 & 0.031 & 0.409 & 0.056 \\
468 & 0.088 & 0.192 & 0.585 \\
469 & 0.103 & 1 & 0.014 \\
470 & 0.761 & 0.500 & 0.094 \\
483 & 1 & 0.562 & 0.086 \\
491 & 0.416 & 0.561 & 0.013 \\
509 & 0.033 & 0.657 & 0.062 \\
520 & 0.097 & 0.002 & 0.482 \\
524 & 0.719 & 0.044 & 1 \\
\hline
\end{tabular}

*Only the p-values of mutations that are either statistical associated or showing the tendency to be associated to N-terminal resistance-associated mutations are shown

*2 All replacements that occurred at the indicated sites were included in the statistic analysis 
Substitutions at one position (399) were highly associated with TAMs. These substitutions were also associated with NAMs.

Substitutions at two other positions (369 and 395) were also associated with TAMs and NAMs. Substitutions at two positions (520 and 524) were only associated with NAMs, although substitutions at position 520 showed the tendency to be associated with TAMs. Substitutions at two positions (451 and 509) were associated with TAMs and showed the tendency to be associated with NNRTI-resistance associated mutations.

Furthermore, substitutions at some positions were only associated with NNRTI-resistance delivering mutations (431, 469 and 491) and there was a trend for substitutions at two positions to be associated with NNRTI-resistance associated mutations (470 and 483).

\section{DisCUSSION}

We identified several substitutions in the connection domain (e.g. N348I, A360V, V365I and T376S), which have earlier been accounted for an increased AZT-resistance and have been identified in-vivo [6, 12, 16, 17]. The identified substitutions were neither associated with an antiviral therapy nor with resistance-associated N-terminal mutations.

Previous studies have shown that especially N348I is prevalent amongst pre-treated patients and causes NRTI- and NNRTI-resistance in-vitro and in-vivo [6, $9,10,12,17,18]$. It is able to restore the reduced RT activity of virus isolates containing the NRTI-resistance delivering mutation M184V [19]. A recent study has shown that N348I may enable the selection of both TAMs and mutations that are antagonistic toward TAMs [20]. N348I evolves prior to TAMs [10, 20]. This could explain the lacking association with TAMs observed in this study. Furthermore, it has been shown that the M184V mutation combined with TAMs is crucial for the development of the N348I mutation because the N384I mutation restores fitness loss due to the M184V in a TAM background [19]. As mentioned above only 16 of the analyzed sequences contained M184V.

We identified another connection domain substitution (E399D) that is associated with a current antiretroviral treatment and NRTI-resistance delivering mutations. E399D has previously been reported to be associated with a significant reduction in etravirine susceptibility [21].

We did not find any of the primer grip substitutions created by Nikolenko et al., which have been shown to increase AZT-resistance [7].

Instead, there were other substitutions, but almost all of them - except for K476Q - were located in the connection domain. This finding is consistent with a recently performed in-vivo study, in which especially the primer grip residues located in the $\mathrm{RNase} H$ were conserved [12]. However, substitutions at these residues have been found in-vivo and in database analyses $[3,11]$. Such mutations lead to an inefficient ppt-primer removal, which could be fatal for viral replication [22]. Another study supports this deliberation, showing that substitutions at position 475 and 501 lead to defective viruses [23].
Only substitutions at one primer grip position (395) were associated with $\mathrm{N}$-terminal mutations. These substitutions also showed the tendency to be associated with an AZT-treatment six month prior to sample taking. K395A was one of the mutations created by Nikolenko et al. that decreased viral replicative capacity only marginally [7]. This might explain why the other primer grip substitutions were not associated with treatment. Those substitutions could be silent mutations with no effect on viral resistance or fitness.

Brehm et al. have performed in vitro AZT-selection experiments in which C-terminal mutations appeared (R358K, A371V, F416Y and Q509L). Especially the combination of A371V, Q509L and TAMs was able to augment AZT-resistance [8].

Similar to other in vivo studies we identified substitutions at position 358, 371 and 509 [11, 12, 17]. None of these substitutions was associated with an antiretroviral treatment, but substitutions at position 358 showed the trend to be associated with TAMs and substitutions at position 509 were associated with TAMs.

Two RNase H mutants (H539N und D549N) created by Nikolenko et al. strongly increase AZT-resistance [3]. Both positions were completely conserved in the analyzed sequences. This is concordant with other in-vivo studies, although mutations at these positions have been found in databases $[11,12,17]$. Such mutations severely reduce viral replication [24-26]. In addition, D549N only increased AZT-resistance in combination with TAMs. Even without TAMs, H539N increased AZT-resistance strongly, but simultaneously it decreased the replication capacity more severely, making its in-vivo appearance much less likely [3].

Different in-vivo studies have described substitutions at RNase $H$ residues (469, 470, 554 and 558) [11, $12,17]$. Although we also identified such substitutions, none of them was associated with treatment except for A554T and the only substitutions associated with $\mathrm{N}$-terminal resistance delivering mutations were substitutions at position 469, although substitutions at position 470 also showed the trend to be associated with NNRTI resistance-delivering mutations. Resistance testing has shown that L469I does not increase drugresistance [17]. Positive effects on viral replication might explain its association with TAMs.

In the present study, viral sequences isolated from HIV-1 infected patients treated at the University Hospital Frankfurt/Main have been analyzed to evaluate the in-vivo presence of C-terminal substitutions, which could cause drug-resistance. Several previously reported substitutions were present; however, more studies to evaluate their phenotypic effects are necessary.

The statistical association of some substitutions with N-terminal resistance-delivering mutations other than TAMs is probably because antiretroviral treatment selects both of them.

Other substitutions were associated with N-terminal-resistance-associated mutations, although there was no correlation to an antiretroviral therapy. An explanation could be that some sequences were derived from patients, who have been primarily infected by a virus from a pre-treated individual. Furthermore, 
some patients have been in a selected treatment interruption at the time of the sampling. A missing selective pressure could lead to an overgrowth of a virus with C-terminal mutations by the drug-sensitive wildtype. Additionally, genotyping can only show mutations with a proportion within the quasispecies higher than 20 percent [27].

A recent study has shown that the combination of two C-terminal mutations (T369I and N348I) reduces susceptibility to NRTIs and NNRTIs [28]. There are only a few other studies, which have considered the phenotypic effects of combinations of C-terminal mutations. There might be other combinations of Cterminal mutations leading to an increased drug resistance.

In conclusion, our results show that some of the Cterminal mutations described in vitro are present in patient isolates. A few of them could be associated with antiviral treatment and drug resistance-associated mutations in the polymerase region.

Due to the low prevalence of C-terminal mutations and because only a few of them could be associated with antiviral treatment and drug resistance-associated mutations in the polymerase region in our study, we would not recommend routinely testing of the C-terminal RT region. If new sequencing techniques like pyrosequencing become more cost effective, adding the C-terminal RT region to routine testing should be reconsidered.

However, due to the low prevalence of these C-terminal substitutions in vivo, larger studies will have to confirm the clinical significance of our findings.

Acknowledgements and conflicts of interest: We would like to thank Ms. Margarethe Podporski for performing the sequencing analysis. All authors have no conflict of interest with this work and none to declare.

\section{REFERENCES}

1. Clavel F, Hance AJ. HIV drug resistance. N Engl J Med. 2004 Mar 4; 350 (10): 1023-35.

2. Meyer PR, Matsuura SE, Mian AM, So AG, Scott WA. A mechanism of AZT resistance: an increase in nucleotidedependent primer unblocking by mutant HIV-1 reverse transcriptase. Mol Cell. 1999 Jul; 4 (1): 35-43.

3. Nikolenko GN, Palmer S, Maldarelli F, Mellors JW, Coffin JM, Pathak VK. Mechanism for nucleoside analogmediated abrogation of HIV-1 replication: balance between RNase $\mathrm{H}$ activity and nucleotide excision. Proc Natl Acad Sci U S A. 2005 Feb 8; 102 (6): 2093-8.

4. Sluis-Cremer N, Arion D, Parniak MA. Molecular mechanisms of HIV-1 resistance to nucleoside reverse transcriptase inhibitors (NRTIs). Cell Mol Life Sci. 2000 Sep; 57 (10): 1408-22.

5. Radzio J, Sluis-Cremer N. Efavirenz accelerates HIV-1 reverse transcriptase ribonuclease $\mathrm{H}$ cleavage, leading to diminished zidovudine excision. Mol Pharmacol. 2008 Feb; 73 (2): 601-6.

6. Nikolenko GN, Delviks-Frankenberry KA, Palmer S, Maldarelli F, Fivash MJ, Jr., Coffin JM, Pathak VK. Mutations in the connection domain of HIV-1 reverse transcriptase increase 3'-azido-3'-deoxythymidine resistance. Proc Natl Acad Sci U S A. 2007 Jan 2; 104 (1): 317-22.

7. Delviks-Frankenberry KA, Nikolenko GN, Barr R, Pathak VK. Mutations in human immunodeficiency virus type 1 RNase $H$ primer grip enhance 3'-azido-3'-de- oxythymidine resistance. J Virol. 2007 Jul; 81 (13): 683745.

8. Brehm JH, Koontz D, Meteer JD, Pathak V, Sluis-Cremer N, Mellors JW. Selection of mutations in the connection and $\mathrm{RNase} \mathrm{H}$ domains of human immunodeficiency virus type 1 reverse transcriptase that increase resistance to 3'-azido-3'-dideoxythymidine. J Virol. 2007 Aug; 81 (15): 7852-9.

9. Hachiya A, Kodama EN, Sarafianos SG, Schuckmann MM, Sakagami Y, Matsuoka M, Takiguchi M, Gatanaga H, Oka S. Amino Acid Mutation N348I in the Connection Subdomain of HIV-1 Reverse Transcriptase Confers Multi-class Resistance to Nucleoside and Non-nucleoside Reverse Transcriptase Inhibitors. J Virol. 2008 Jan 23; 82 (7): 3261-70.

10. Yap SH, Sheen CW, Fahey J, Zanin M, Tyssen D, Lima VD, Wynhoven B, Kuiper M, Sluis-Cremer N, Harrigan PR, Tachedjian G. N348I in the connection domain of HIV-1 reverse transcriptase confers zidovudine and nevirapine resistance. PLoS Med. 2007 Dec; 4 (12): e335.

11. Roquebert B, Wirden M, Simon A, Deval J, Katlama C, Calvez V, Marcelin AG. Relationship between mutations in HIV-1 RNase $\mathrm{H}$ domain and nucleoside reverse transcriptase inhibitors resistance mutations in naive and pretreated HIV infected patients. J Med Virol. 2007 Mar; 79 (3): 207-11.

12. Santos AF, Lengruber RB, Soares EA, Jere A, Sprinz E, Martinez AM, Silveira J, Sion FS, Pathak VK, Soares MA. Conservation patterns of HIV-1 RT connection and RNase $\mathrm{H}$ domains: identification of new mutations in NRTI-treated patients. PLoS ONE. 20083 (3): e1781.

13. Sturmer M, Berger A, Doerr HW. Modifications and substitutions of the RNA extraction module in the ViroSeq HIV-1 genotyping system version 2: effects on sensitivity and complexity of the assay. J Med Virol. 2003 Dec; 71 (4): 475-9.

14. Stürmer M, Berger A, Preiser W. HIV-1 genotyping: comparison of two commercially available assays. Expert Rev Mol Diagn. 2004 May; 4 (3): 281-91.

15. Sturmer M, Zimmermann K, Fritzsche C, Reisinger E, Doelken G, Berger A, Doerr HW, Eberle J, Gurtler LG. Regional spread of HIV-1 M subtype B in middle-aged patients by random env-C2V4 region sequencing. Med Microbiol Immunol. 2010 Mar; 199(2): 123-8.

16. Delviks-Frankenberry KA, Nikolenko GN, Boyer PL, Hughes SH, Coffin JM, Jere A, Pathak VK. HIV-1 reverse transcriptase connection subdomain mutations reduce template RNA degradation and enhance AZT excision. Proc Natl Acad Sci U S A. 2008 Aug 5; 105 (31): 10943-8.

17. Ntemgwa M, Wainberg MA, Oliveira M, Moisi D, Lalonde R, Micheli V, Brenner BG. Variations in reverse transcriptase and RNase $\mathrm{H}$ domain mutations in human immunodeficiency virus type 1 clinical isolates are associated with divergent phenotypic resistance to zidovudine. Antimicrob Agents Chemother. 2007 Nov; 51 (11): 3861-9.

18. Ehteshami M, Beilhartz GL, Scarth BJ, Tchesnokov EP, McCormick S, Wynhoven B, Harrigan PR, Gotte M. Connection domain mutations N348I and A360V in HIV-1 reverse transcriptase enhance resistance to 3 '-azido-3'-deoxythymidine through both RNase H-dependent and -independent mechanisms. J Biol Chem. 2008 Aug 8; 283 (32): 22222-32.

19. von Wyl V, Ehteshami M, Symons J, Burgisser P, Nijhuis M, Demeter LM, Yerly S, Boni J, Klimkait T, Schuurman R, Ledergerber B, Gotte M, Gunthard HF. Epidemiological and Biological Evidence for a Compensatory Effect of Connection Domain Mutation N348I on M184V in HIV1 Reverse Transcriptase. J Infect Dis. 2010 Apr 1; 201 (7): 1054-1062.

20. Radzio J, Yap SH, Tachedjian G, Sluis-Cremer N. N348I 
in reverse transcriptase provides a genetic pathway for HIV-1 to select thymidine analogue mutations and mutations antagonistic to thymidine analogue mutations. Aids. 2010 Mar; 24(5): 659-67.

21. Poveda E, de Mendoza C, Pattery T, Gonzalez Mdel M, Villacian J, Soriano V. Phenotypic impact of resistance mutations on etravirine susceptibility in HIV patients with prior failure to nonnucleoside analogues. Aids. 2008 Nov 12; 22 (17): 2395-8.

22. Julias JG, McWilliams MJ, Sarafianos SG, Arnold E, Hughes SH. Mutations in the RNase H domain of HIV-1 reverse transcriptase affect the initiation of DNA synthesis and the specificity of RNase $\mathrm{H}$ cleavage in vivo. Proc Natl Acad Sci U S A. 2002 Jul 9; 99 (14): 9515-20.

23. Arion D, Sluis-Cremer N, Min KL, Abram ME, Fletcher RS, Parniak MA. Mutational analysis of Tyr-501 of HIV-1 reverse transcriptase. Effects on ribonuclease $\mathrm{H}$ activity and inhibition of this activity by $\mathrm{N}$-acylhydrazones. J Biol Chem. 2002 Jan 11; 277 (2): 1370-4.

24. Tisdale M, Schulze T, Larder BA, Moelling K. Mutations within the RNase $\mathrm{H}$ domain of human immunodeficiency virus type 1 reverse transcriptase abolish virus infectivity. J Gen Virol. 1991 Jan; 72: 59-66.

25. Cristofaro JV, Rausch JW, Le Grice SF, DeStefano JJ. Mutations in the ribonuclease $\mathrm{H}$ active site of HIV-RT reveal a role for this site in stabilizing enzyme-primertemplate binding. Biochemistry. 2002 Sep 10; 41 (36): 10968-75.

26. Volkmann S, Wohrl BM, Tisdale M, Moelling K. Enzymatic analysis of two HIV-1 reverse transcriptase mutants with mutations in carboxyl-terminal amino acid residues conserved among retroviral ribonucleases H. J Biol Chem. 1993 Feb 5; 268 (4): 2674-83.
27. Larder BA, Kohli A, Kellam P, Kemp SD, Kronick M, Henfrey RD. Quantitative detection of HIV-1 drug resistance mutations by automated DNA sequencing. Nature. 1993 Oct 14; 365 (6447): 671-3.

28. Gupta S, Fransen S, Paxinos EE, Stawiski E, Huang W, Petropoulos CJ. Combinations of Mutations in the Connection Domain of Human Immunodeficiency Virus Type 1 Reverse Transcriptase: Assessing the Impact on Nucleoside and Non-Nucleoside Reverse Transcriptase Inhibitor Resistance. Antimicrob Agents Chemother. 2010 May; 54(5): 1973-80.

Received: July 14, 2010 / Accepted: August 20, 2010

Address for correspondence:

Martin Stürmer

J.W. Goethe University Hospital

Institute for Med. Virology

Paul Ehrlich Str. 40

60596 Frankfurt

Germany

Phone: +496963016454

Fax: $\quad+496963016477$

E-mail: M.Stuermer@em.uni-frankfurt.de 